\title{
Apoptosis is induced by docosahexaenoic acid in breast cancer cells via death receptor and mitochondria-mediated pathways
}

\author{
MEILAN XUE ${ }^{1}$, YINLIN GE ${ }^{1}$, CHUNDONG YU $^{2}$, ZHENG ZHENG $^{1}$, XINJIA HE $^{3}$ and JINGLAN ZHAO ${ }^{4}$ \\ ${ }^{1}$ Department of Biochemistry and Molecular Biology, Medical College, Qingdao University, Qingdao, Shandong 266021; \\ ${ }^{2}$ Department of Laboratory, Women and Children's Hospital of Qingdao, Qingdao, Shandong 266034; \\ ${ }^{3}$ Department of Oncology, The Affiliated Hospital of Qingdao University, Qingdao, Shandong 266003; \\ ${ }^{4}$ Department of Cardiothoracic Surgery, Qingdao Center Medical Group, Qingdao, Shandong 266033, P.R. China
}

Received May 6, 2016; Accepted April 4, 2017

DOI: $10.3892 / \mathrm{mmr} .2017 .6678$

\begin{abstract}
In the present study, the antitumor effect of n-3 fatty acid was evaluated, and the effect of docosahexaenoic acid (DHA) on the induction of apoptosis and its underlying mechanism were examined. Flow cytometry and western blot analysis were performed to analyze apoptosis and the expression of protein factors in human breast cancer cells. The data revealed that DHA inhibited the viability of MCF-7 breast cancer cells in vitro, and promoted cell death by the induction of apoptosis. DHA decreased the expression of B-cell lymphoma 2 (Bcl-2), whereas the expression of $\mathrm{Bcl}-2$-associated $\mathrm{X}$ protein was increased. DHA was also shown to promote the release of Smac/Diablo and cytochrome $c$ from the mitochondria. DHA increased the levels of cleaved caspase-8, -9 and -3 . Additionally, the protein expression of tumor necrosis factor-related apoptosis-inducing ligand, death receptor 4 and Fas were increased following DHA treatment. In conclusion, DHA caused apoptosis of the human breast cancer cells in vitro through the death receptor and mitochondria-mediated pathways. The results of this study encourage further investigation of the effect of fish oil on the prevention and treatment of human breast cancer.
\end{abstract}

\section{Introduction}

The n-3 and n- 6 fatty acids (FAs) are essential and important to human health. However, there is a disproportionally high proportion of n-6 FAs and low proportion of n-3 FAs in our current dietary structure. This results in a high ratio of $n-6 / n-3$, which is associated with cardiovascular disease, inflammation and cancer (1-3). The n-3 FAs are long-chain polyunsaturated

Correspondence to: Dr Yinlin Ge, Department of Biochemistry and Molecular Biology, Medical College, Qingdao University, 38 Dengzhou Road, Qingdao, Shandong 266021, P.R. China E-mail: geyinlin57@163.com

Key words: docosahexaenoic acid, breast cancer, apoptosis, Fas, death receptor
FAs. Eicosapentaenoic acid (EPA; 20:5n-3) and docosahexaenoic acid (DHA; 22:6n-3) are two important n-3 FAs. The primary dietary source of these is oily fish (4). Investigations in human populations have revealed that a high consumption of fish or fish oils can decrease the risk of breast, prostate and colon cancer $(5,6)$. Previous laboratory experiments have shown that n-3 FAs can inhibit the initiation and development of breast cancer in vivo $(7,8)$, although others have failed to identify a significant association.

The n-3 FAs have a number of biological effects, and effects of n-3 FAs on cancer suppression have been suggested $(9,10)$, with n-3 FAs affecting the apoptosis, proliferation, invasion and metastasis of cancer cells. There are several signaling pathways involved in the effects of n-3 FAs, including protein kinase C, extracellular signal-regulated kinase (ERK) 1/2, ras and nuclear factor $(\mathrm{NF})-\kappa \mathrm{B}(11-14)$. However, the effect of n-3 FAs on the death receptor-mediated pathways and its molecular mechanisms remain to be fully elucidated.

In the present study, the antitumor effect of n-3 FAs was investigated, with a focus on the apoptosis-inducing effects of DHA on MCF-7 human breast cancer cells in culture. An improved understanding of the mechanism underlying the antitumor effects of n-3 FAs may assist in developing novel tumor treatment strategies involving the use of fish oil as a dietary supplement.

\section{Materials and methods}

Chemicals and reagents. DHA, EPA, linoleic acid (18:2n-6) and arachidonic acid (20:4n-6) in fetal bovine serum (FBS) were purchased from Sigma-Aldrich; Merck Millipore (Darmstadt, Germany). Anti- $\beta$-actin antibody and horseradish peroxidase (HRP)-conjugated anti-rabbit, anti-mouse and anti-goat IgG were obtained from Amersham; GE Healthcare Life Sciences (Chalfont, UK; 600567, RPN4301, RPN4201 and PA42002). Antibodies against cytochrome $c$ and tumor necrosis factor-related apoptosis-inducing ligand (TRAIL) were obtained from BD Pharmingen (Franklin Lake, NJ, USA; 558700 and 556468). The antibodies against cleaved caspase-3, cleaved caspase- 8 and cleaved caspase- 9 were obtained from Cell Signaling Technology, Inc. (Beverly, MA, USA; 9661S, 9496S and 9501S). Antibodies against death 
receptor (DR) 4 and 5 were purchased from Imgenex; Novus Biologicals LLC (Littleton, CO, USA; NB100-56747 and NB-100-55744). Antibodies against B-cell lymphoma 2 (Bcl-2), Bcl-2-associated X protein (Bax), Fas, Fas ligand (FasL) and Smac/Diablo were purchased from Santa Cruz Biotechnology, Inc. (Dallas, TX, USA; sc-509, sc-7480, sc-8009, sc-33716 and sc-393118).

Cell culture. The MCF-7 breast cancer cells were purchased from Shanghai Life Science of Chinese Academy of Sciences (Shanghai, China). The MCF-7 cells were cultured in Roswell Park Memorial Institute 1640 medium (Hyclone; GE Healthcare Life Sciences, Logan, UT, USA), supplemented with 10\% heat-inactivated FBS (Gibco; Thermo Fisher Scientific, Inc., Waltham, MA, USA), in an incubator at $37^{\circ} \mathrm{C}$ with $5 \% \mathrm{CO}_{2}$ and $98 \%$ relative humidity. The MCF-7 cells were seeded in 6-well plates in routine cultivation. Cells in the exponential growth phrase were used in the subsequent experiments.

\section{3-(4,5-dimethylthiazole-2-yl)-2,5-diphenyl tetrazolium} bromide (MTT) assay. The MTT (Roche Diagnostics, Basel, Switzerland) assay was performed as previously described (15). The MCF-7 cells $\left(1 \times 10^{4}\right.$ cells per well) were seeded in triplicate on a 96-well plate and cultured overnight prior to treatment with 25, 50 and $100 \mu \mathrm{M}$ DHA or EPA for 24 or $72 \mathrm{~h}$. Following incubation, MTT dye was added and the mixture was incubated for $4 \mathrm{~h}$ at $37^{\circ} \mathrm{C}$. The supernatant was removed and $150 \mu \mathrm{l}$ DMSO was added to each well to dissolve the crystals completely, following which an ELISA reader (Bio-Rad Laboratories, Inc., Hercules, CA, USA) was used to determine the absorbance at $490 \mathrm{~nm}$. The results are expressed as the percentage of inhibition, which led to a reduction in absorbance by DHA, compared with that in the control group.

Analysis of apoptosis using flow cytometry. An Annexin V-FITC apoptosis detection kit was used for the analysis of apoptosis (Invitrogen; Thermo Fisher Scientific, Inc.). DHA concentrations of 25,50 and $100 \mu \mathrm{M}$ were used to treat the MCF-7 cells $\left(1 \times 10^{6}\right.$ cells $\left./ \mathrm{ml}\right)$, respectively, for $48 \mathrm{~h}$ at $37^{\circ} \mathrm{C}$. The cells were digested in trypsin, washed twice with PBS and resuspended in $500 \mu \mathrm{l}$ binding buffer. The cell suspensions were then treated with $5 \mu \mathrm{l}$ of Annexin V-FITC and $5 \mu \mathrm{l}$ propidium iodide (PI). Following treatment for $10 \mathrm{~min}$ at room temperature, the apoptosis of the cells were determined immediately using flow cytometry (BD Biosciences, Franklin Lakes, NJ, USA).

Analysis of proteins using western blot analysis. The MCF-7 cells were grown on 6 -well plates $\left(1 \times 10^{6}\right.$ cells $\left./ \mathrm{ml}\right)$, to which 25, 50 and $100 \mu \mathrm{M}$ DHA were added and incubated for $48 \mathrm{~h}$. The cells were lysed in $0.5 \mathrm{ml}$ lysis buffer (PBS containing $1 \%$ Triton $\mathrm{X}-100$ and $1 \mathrm{mM}$ PMSF) at $4^{\circ} \mathrm{C}$ for $10 \mathrm{~min}$. The concentrations of protein were then measured using a BCA assay. For western blot analysis, cell lysates $(5 \mu \mathrm{g})$ from the cancer cell culture were subjected to $12 \%$ SDS-PAGE, transferred to polyvinylidene difluoride membranes, and blocked with 5\% nonfat milk in TBS-Tween buffer (TBST; 20-mM Tris-HCl, $120 \mathrm{mMNaCl}, 0.1 \%$ Tween-20) for 1 has described previously (16). Membranes were incubated with primary antibodies against cleaved caspase-3, -8 -9 (dilution, 1:500), Bcl-2,

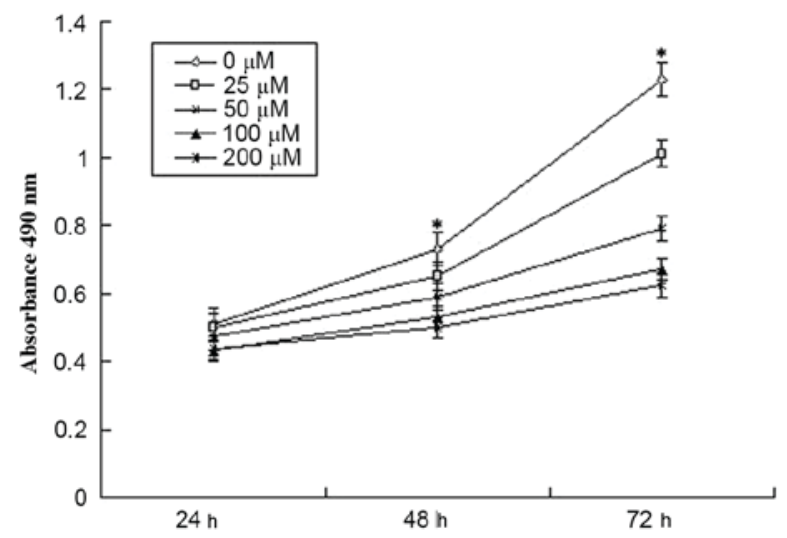

Figure 1. Inhibitory effect of DHA on the growth of MCF-7 cells. The effect of DHA on the viability of cultured MCF-7 cells was determined using a3-(4,5-dimethylthiazole-2-yl)-2,5-diphenyl tetrazolium bromide assay. Treatment with 25,50, 100 and $200 \mu \mathrm{M}$ DHA reduced the viability of the MCF-7 cells $(\mathrm{P}<0.05)$. Following treatment with $100 \mu \mathrm{M}$ DHA for $72 \mathrm{~h}$, the proliferation rate of the MCF-7 cells was inhibited by $45.5 \% .{ }^{*} \mathrm{P}<0.05$, vs. control. DHA, docosahexaenoic acid.

Bax, Fas, FasL (dilution, 1:1,000), Smac (dilution, 1:400), DR4 and DR5 (dilution, 1:200) at $4^{\circ} \mathrm{C}$ overnight. Following 3 washes ( 5 min each) with TBST, the membranes were incubated with an appropriate secondary antibody for $2 \mathrm{~h}$ at room temperature. Membranes were washed 3 times (5 min each) with TBST, and protein expression was quantified using a Gel EDAS analysis system (Cold Spring USA Corporation, Cherry Hill, NJ, USA) and Gel-Pro Analyzer 3.1 software (Media Cybernetics, Inc., Rockville, MD, USA).

Statistical analysis. Statistical analyses were performed using one-way analysis of variance with SPSS 11.0 software (SPSS, Inc., Chicago, IL, USA). Comparison between groups was performed using Duncan's test. All data are presented as the mean \pm standard deviation. $\mathrm{P}<0.05$ was considered to indicate a statistically significant difference.

\section{Results}

Inhibitory effect of DHA on the growth of breast cancer cells. The effect of DHA on the viability of MCF-7 cells was initially determined using an MTT assay. As shown in Fig. 1, DHA treatment decreased the viability of the MCF-7 cells $(\mathrm{P}<0.05)$. Following treatment with $100 \mu \mathrm{M}$ DHA for $72 \mathrm{~h}$, the proliferation of the MCF-7 cells was inhibited by $45.5 \%$.

DHA induces apoptosis of breast cancer cells. The apoptosis of cells was estimated using flow cytometry. The apoptotic rates were higher in the DHA-treated cells, compared with that in the control (Fig. 2). Following treatment with DHA (25, 50 and $100 \mu \mathrm{M}$ ) for $48 \mathrm{~h}$, the proportions of early apoptotic MCF-7 cells were 26.7, 38.1 and 50.6\%, respectively $(\mathrm{P}<0.05)$.

Effect of DHA on the expression of the Bcl-2 family proteins. The increased expression of Bax is reported as an apoptosis-signaling pathway in breast cancer cells and proteins of the Bcl-2 family are important factors in apoptosis regulation (17-19). In the present study, the expression levels of Bcl-2 
A

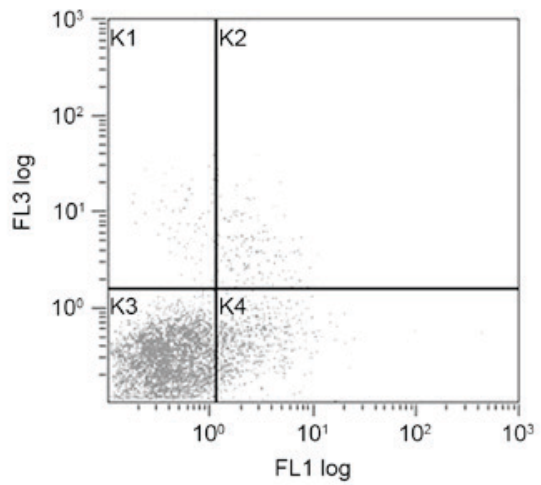

C

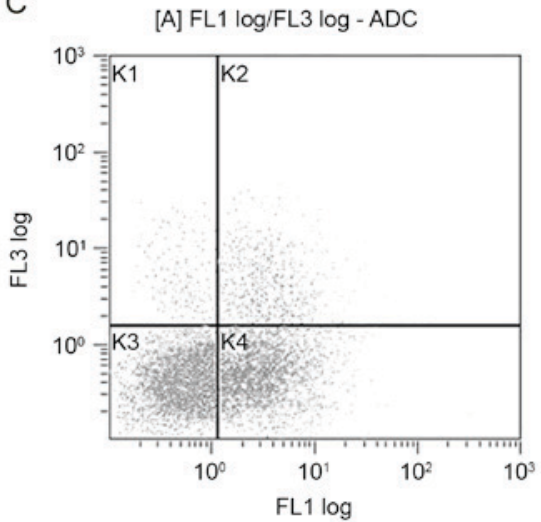

B [A] FL1 log/FL3 log - ADC

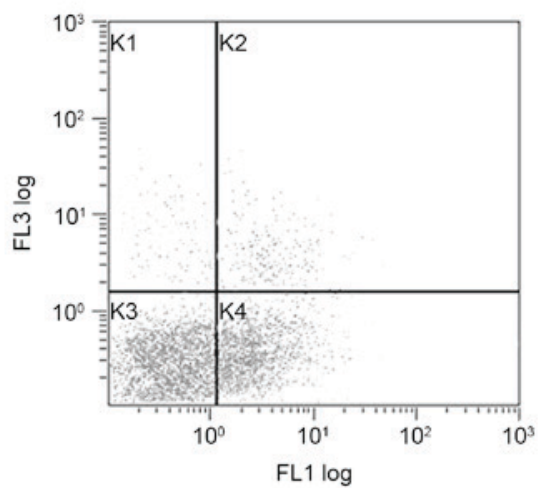

D

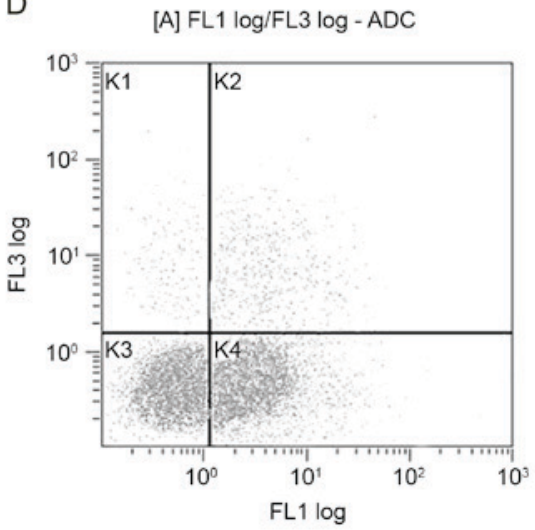

Figure 2. Flow cytometric analysis of the apoptosis of MCF-7 cells treated with DHA for $48 \mathrm{~h}$. (A) Control; (B) $25 \mu \mathrm{M}$ DHA; (C) $50 \mu \mathrm{M}$ DHA; (D) $100 \mu \mathrm{M}$ DHA. MCF-7 cells were stained with Annexin V-FITC and propidium iodide. Following treatment with DHA, the early apoptotic rates of the MCF-7 cells were 26.7, 38.1 and 50.6\% respectively, and were significantly increased, compared with that in the untreated cells $(\mathrm{P}<0.05)$. The gated quadrants were as follows: K1, Annexin V-/PI+, necrosis; K2, Annexin V+/PI+, late apoptosis and necrosis; K3, Annexin V-/PI-, normal cells without apoptosis or necrosis; K4, Annexin V+/PI-, early apoptosis. DHA, docosahexaenoic acid.

A
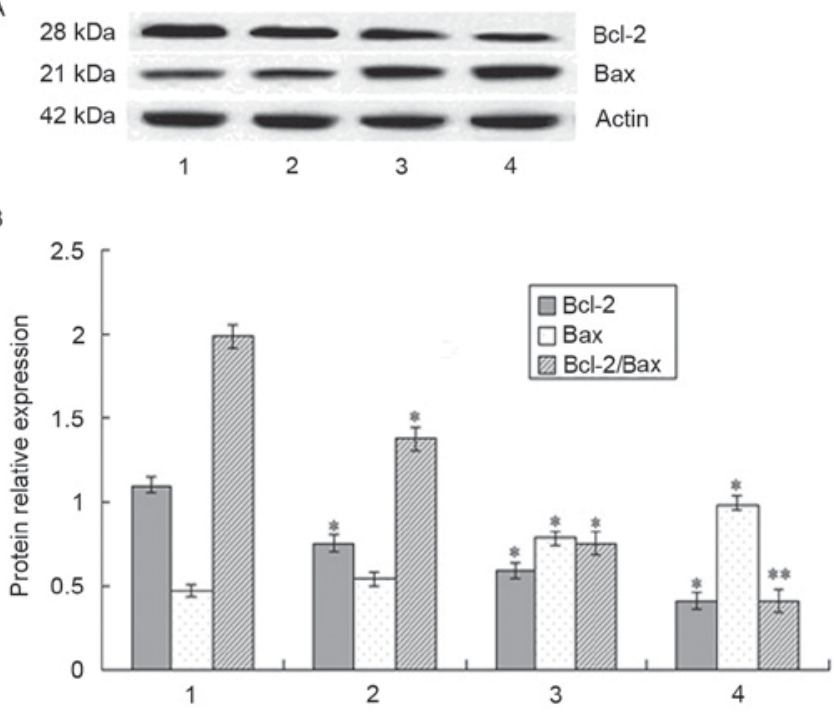

Figure 3. Expression of Bcl-2 and Bax in MCF-7 cells following treatment with DHA for $48 \mathrm{~h}$, detected using western blot analysis. (A) Protein levels of Bcl-2 and Bax were measured using western blot analysis. Expression of $\beta$-actin was used as the internal control. (B) Relative expression levels of $\mathrm{Bcl}-2$ and Bax and the expression ratio of the two are shown. DHA reduced the level of Bcl-2, increased the level of Bax and reduced the Bcl-2/Bax ratio. Lane 1, control cells; lanes 2-4, MCF-7 cells treated with 50, 100 and $200 \mu$ M DHA, respectively. "P $<0.05$ and ${ }^{* *} \mathrm{P}<0.01$, vs. control. Bcl-2, B-cel lymphoma 2; Bax, Bcl-2-associated X protein; DHA, docosahexaenoic acid. and Bax were examined in MCF-7 cells following treatment with DHA. The expression of Bcl-2 was reduced and the expression of Bax was increased following DHA treatment. Therefore, the ratio of Bcl-2/Bax was decreased (Fig. 3).

DHA increases the activation of caspases. Caspases are central effectors in apoptosis. In order to identify the mechanisms responsible for DHA-induced apoptosis, the present study detected the levels of activated caspases using western blot analysis to identify the mechanism underlying the induction of apoptosis by DHA. Following treatment of the cells with DHA, the levels of cleavedcaspases- $8,-9$ and -3 were increased (Fig. 4).

DHA induces the mitochondria to release cytochrome $c$ and Smac/Diablo. In apoptosis, mitochondria release cytosolic cytochrome $c$ and Smac/Diablo, which then activate caspase-9 (20). As DHA increased the activation of caspase-9, the levels of cytochrome $c$ and Smac/Diablo in the cytoplasm were determined in the present study. As shown in Fig. 5, DHA treatment markedly increased the levels of cytoplasmic cytochrome $c$ and Smac/Diablo. This suggested that DHA induced the mitochondria to release cytochrome $c$ and Smac/Diablo.

DHA increases the expression of death receptors. Caspase- 8 is involved in the death receptor-mediated apoptotic pathway. 


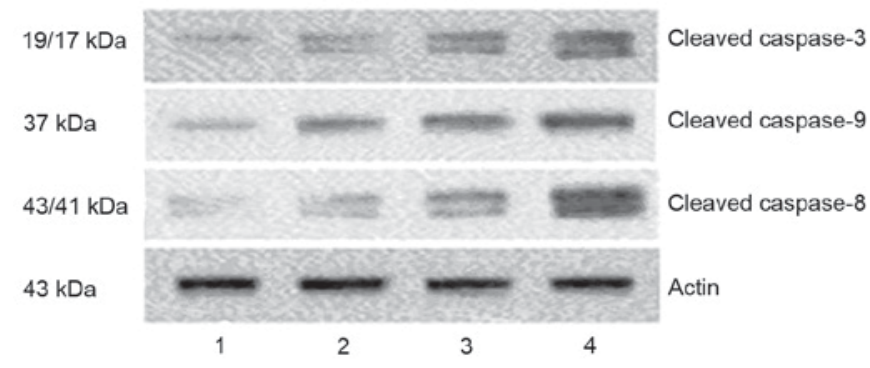

Figure 4. DHA induces increases in the levels of cleaved caspases in MCF-7 cells. Following treatment with DHA for 48 h, MCF-7 cell lysates were detected using western blot analysis. Images representative of three independent experiments are shown. The levels of cleaved caspases-8, -9 and -3 were increased following DNA treatment. Lane 1, control cells; lanes 2-4 MCF-7 cells treated with 50, 100 and $200 \mu \mathrm{M}$ DHA, respectively. DHA, docosahexaenoic acid.

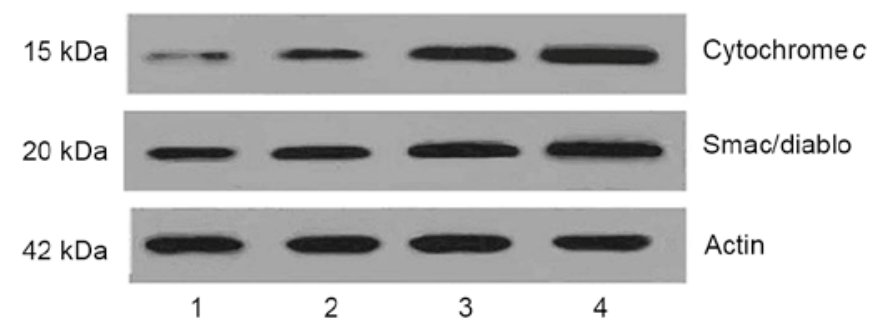

Figure 5. DHA increases the release of cytochrome $c$ and Smac/Diablo from the mitochondria in MCF-7 cells. Cells were treated with 50, 100 and $200 \mu \mathrm{M}$ DHA, respectively, for $48 \mathrm{~h}$, then treated with subcellular fractionation. The levels of cytochrome $c$ and Smac/Diablo in the resultant cytosolic fractions were detected using western blot analysis. Following treatment with DHA the levels of cytochrome $c$ and Smac/Diablo in the cytoplasm were increased. Lane 1, control cells; lanes 2-4, MCF-7 cells treated with 50, 100 and $200 \mu \mathrm{M}$ DHA, respectively. DHA, docosahexaenoic acid.

In the present study, DHA treatment increased the level of activated caspase-8; therefore, the levels of death receptors and membrane-bound death receptor ligands were determined. Following DHA intervention, the expression levels of Fas, DR4, and TRAIL were increased, whereas no significant changes in the expression levels of FasL or DR5were observed (Fig. 6).

\section{Discussion}

In the present study, it was found that DHA, an n-3FA, resulted in the inhibition of cell viability and the induction of cell apoptosis. These results confirmed and extended earlier observations (21-23) on the antitumor effect of n-3 FAs.

The results of previous studies revealed that the antitumor effect of DHA is due to the induction of apoptosis (21-23). There are also reports showing that EPA treatment of HL-60 cells induces the activation of caspase- $3,-6,-8$ and -9 , and the release of cytochrome $c$ from mitochondria (24). In the present study, the results of the flow cytometry confirmed that DHA treatment induced apoptosis of the breast cancer cells.

The exact role of n-3 FAs in the development, progression and prevention of breast cancer remains to be fully elucidated. The n-3 FAs are involved in the development and progression of tumors via multiple mechanisms. Several signaling pathways are associated with carcinogenesis and

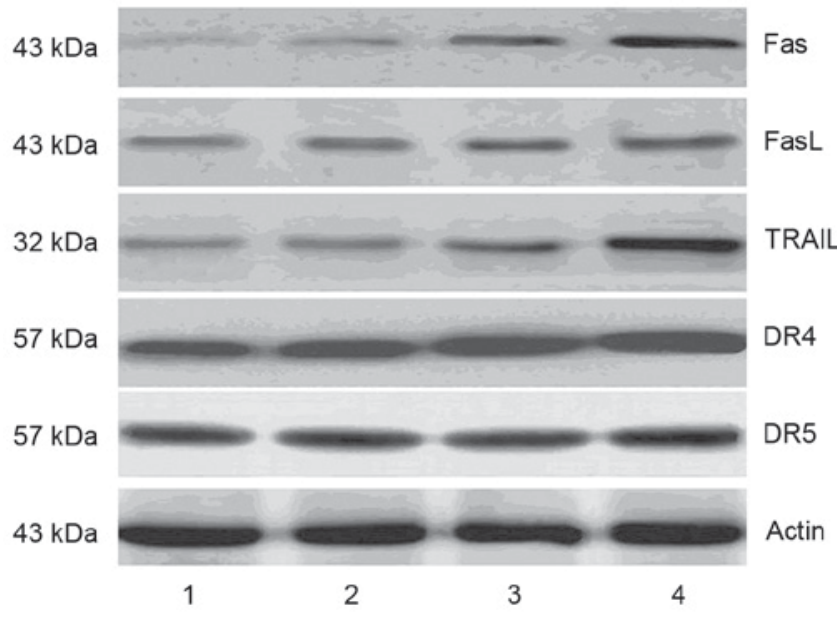

Figure 6. DHA increases the expression of cell death receptors and their ligands in MCF-7 cells. Following treatment with different concentrations of DHA, the protein levels of Fas, FasL, DR4, DR5 and TRAIL were measured using western blot analysis. The results showed that DHA treatment increased the expression levels of Fas, DR4, and TRAIL, whereas the expression levels of FasL and DR5 remained unchanged. Lane 1, control cells; lanes 2-4, MCF-7 cells treated with 50, 100 and $200 \mu \mathrm{M}$ DHA, respectively. DHA, docosahexaenoic acid; FasL, Fas ligand; DR, death receptor; TRAIL, tumor necrosis factor-related apoptosis-inducing ligand.

tumor progression by n-3 polyunsaturated FAs. For example, n-3FAscan downregulate and inactivate cellular signaling mediators, including protein kinase $\mathrm{C}$, ras, ERK1/2, and $\mathrm{NF}-\kappa \mathrm{B}$ (11-14). In addition, n-3 FAs can affect prostate inflammation and carcinogenesis via the cyclooxygenase (COX)-2 enzymatic pathway (25). Kang et al (26) revealed that apoptosis was induced by DHA treatment in MCF-7 cells, and that DHA promoted the formation of reactive oxygen species (ROS) and activation of caspase-8. Previous studies have also suggested that DHA can improve the prognosis of patients with breast cancer following chemotherapy through increasing the formation of ROS (27). Dimri et al (28) found that dietary n-3 FAs suppressed the expression of enhancer of zeste homologue 2 (EZH2), and downregulated the expression of E-cadherin and insulin-like growth factor binding protein 3, which are targets of EZH2 in breast cancer cells. The other possible mechanism was associated with the inhibitory effect of n-3 FAs on the expression of COX, the p21 gene and the p53 gene (29). DHA and EPA have also been reported to downregulate cell surface expression of C-X-C chemokine receptor type 4 (CXCR4) and markedly decrease CXCR4-mediated cell migration in breast cells in vitro (30). The exact molecular mechanism underlying this signaling remains to be fully elucidated. Previously, it was reported that the proteins of the Bcl-2 family may be important in n-3 FA-induced cell death. In the present study, data revealed that DHA at concentrations of $25-100 \mu \mathrm{M}$ increased the expression of Bax but reduced the expression of Bcl-2, increased the release of cytochrome $c$ and Smac/Diablo from mitochondria, promoted the activation of caspases, and increased the levels of Fas, DR4 and TRAIL in MCF-7 breast cancer cells. These results suggested that DHA activated caspases and induced apoptosis through the mitochondria-mediated and death receptor-mediated apoptotic pathways.

The results of the present study indicated that further investigations are required to determine whether the 
supplementation of dietary n-3 FAs has an inhibitory effect on tumor growth in patients with breast cancer.

\section{Acknowledgements}

The present study was completed with the support of the Youth Science Fund Project of the Natural Science Foundation of China (grant no. 81502298), the Shandong Provincial Natural Science Foundation (grant nos. ZR2014JL056 and ZR2010CM010), the Development Project of Shandong Province Medical Science and Technology (2013WS0262), Qingdao Postdoctoral Application Research Project (grant no. 2015165), the Ministry of Education Key Laboratory Open Foundation of Marine Culture of China Ocean University (grant no. 2009001), and the Qingdao University Medical College Young Teachers Cultivation Project (grant no. 600201304).

\section{References}

1. Umemoto N, Ishii H, Kamoi D, Aoyama T, Sakakibara T, Takahashi H, Tanaka A, Yasuda Y, Suzuki S, Matsubara T and Murohara T: Reverse association of omega-3/omega- 6 polyunsaturated fatty acids ratios with carotid atherosclerosis in patients on hemodialysis. Atherosclerosis 249: 65-69, 2016.

2. Molfino A, Gioia G, Rossi Fanelli F and Muscaritoli M: The role for dietary omega-3 fatty acids supplementation in older adults. Nutrients 6: 4058-4073, 2014.

3. Gomez-Candela C, Roldan Puchalt MC, Palma Milla S, Lopez Plaza B and Bermejo L: The role of omega-3 fatty acids in diets. J Am Coll Nutr 34 (Suppl 1): S42-S47, 2015.

4. Bartram HP, Gostner A, Scheppach W, Reddy BS, Rao CV, Dusel G, Richter F, Richter A and Kasper H: Effects of fish oil on rectal cell proliferation, mucosal fatty acids, and prostaglandin E2 release in healthy subjects. Gastroenterology 105: 1317-1322, 1993.

5. Liu J and Ma DW: The role of $n-3$ polyunsaturated fatty acids in the prevention and treatment of breast cancer. Nutrients 6 : $5184-5223,2014$

6. Laviano A, Rianda S, Molfino A and Fanelli FR: Omega-3 fatty acids in cancer. Curr Opin Clin Nutr Metab Care 16: 156-161, 2013.

7. Zhang F, Chen Y, Long J, Dong L, Wang Y and Chen Y: Effect of n-3 and n-6 polyunsaturated fatty acids on lipid metabolic genes and estrogen receptor expression in MCF-7 breast cancer cells. Clin Lab 61: 397-403, 2015.

8. Xue M, Wang Q, Zhao J, Dong L, Ge Y, Hou L, Liu Y and Zheng Z: Docosahexaenoic acid inhibited the Wnt/ $\beta$-catenin pathway and suppressed breast cancer cells in vitro and in vivo. J Nutr Biochem 25: 104-110, 2014.

9. Larsson SC, Kumlin M, Ingelman-Sundberg M and Wolk A: Dietary long-chain n-3 fatty acids for the prevention of cancer: A review of potential mechanisms. Am J Clin Nutr 79: 935-945, 2004.

10. Chapkin RS, McMurray DN and Lupton JR: Colon cancer, fatty acids and anti-inflammatory compounds. Curr Opin Gastroenterol 23: 48-54, 2007.

11. McCarty MF: Fish oil may impede tumour angiogenesis and invasiveness by down-regulating protein kinase $\mathrm{C}$ and modulating eicosanoid production. Med Hypotheses 46: 107-115, 1996.

12. Collett ED, Davidson LA, Fan YY, Lupton JR and Chapkin RS: N-6 and n-3 polyunsaturated fatty acids differentially modulate oncogenic Ras activation in colonocytes. Am J Physiol Cell Physiol 280: C1066-C1075, 2001.

13. Sauer LA, Blask DE and Dauchy RT: Dietary factors and growth and metabolism in experimental tumors. J Nutr Biochem 18: 637-649, 2007.
14. Cavazos DA, Price RS, Apte SS and deGraffenried LA: Docosahexaenoic acid selectively induces human prostate cancer cell sensitivity to oxidative stress through modulation of $\mathrm{NF}-\kappa \mathrm{B}$. Prostate 71: 1420-1428, 2001.

15. Carmichael J, DeGraff WG, Gazdar AF, Minna JD and Mitchell JB: Evaluation of a tetrazolium-based semiautomated colorimetric assay: Assessment of radiosensitivity. Cancer Res 47: 943-946, 1987.

16. Cho HJ, Kim WK, Kim EJ, Jung KC, Park S, Lee HS, Tyner AL and Park JH: Conjugated linoleic acid inhibits cell proliferation and ErbB3 signaling in HT-29 human colon cell line. Am J Physiol Gastrointest Liver Physiol 284: G996-G1005, 2003.

17. Choudhuri T, Pal S, Agwarwal ML, Das T and Sa G: Curcumin induces apoptosis in human breast cancer cells through p53-dependent Bax induction. FEBS Lett 512: 334-340, 2002.

18. Bruey JM, Bruey-Sedano N, Luciano F, Zhai D, Balpai R, Xu C, Kress CL, Bailly-Maitre B, Li X, Osterman A, et al: Bcl-2 and $\mathrm{Bcl}-\mathrm{XL}$ regulate proinflammatory caspase-1 activation by interaction with NALP1. Cell 129: 45-56, 2007.

19. Cho MY, Park SY, Park S, Lee YR, Han GD and Kim JA: Geranyl derivative of phloroacetophenone induces cancer cell-specific apoptosis through Bax-mediated mitochondrial pathway in MCF-7 human breast cancer cells. Biol Pharm Bull 35: 98-104, 2012.

20. Li P, Nijhawan D, Budihardjo I, Srinivasula SM, Ahmad M, Alnemri ES and Wang X: Cytochrome $c$ and dATP-dependent formation of Apaf-1/caspase-9 complex initiates an apoptotic protease cascade. Cell 91: 479-489, 1997.

21. Sun H, Berquin IM, Owens RT, O'Flaherty JT and Edwards IJ: Peroxisome proliferator-activated receptor gamma-mediated up-regulation of syndecan- 1 by $n-3$ fatty acids promotes apoptosis of human breast cancer cells. Cancer Res 68: 2912-2919, 2008.

22. Sauer LA, Dauchy RT, Blask DE, Krause JA, Davidson LK and Dauchy EM: Eicosapentaenoic acid suppresses cell proliferation in MCF-7 human breast cancer xenografts in nude rats via a pertussis toxin-sensitive signal transduction pathway. J Nutr 135: 2124-2129, 2005.

23. Siddiqui RA, Jenski LJ, Neff K, Harvey K, Kovacs RJ and Stillwell W: Docosahexanoic acid induces apoptosis in Jurkat cells by a protein phosphatase-mediated process. Biochim Biophys Acta 1499: 265-275, 2001.

24. Arita K, Yamamoto Y, Takehara Y, Utsumi T, Kanno T, Miyaguchi C, Akiyama J, Yoshioka T and Utsumi K: Mechanisms of enhanced apoptosis in HL-60 cells by UV-irradiated n-3 and n-6 polyunsaturated fatty acids. Free Radic Biol Med 35: $189-199,2003$.

25. Reese AC, Fradet V and Witte JS: Omega-3 fatty acids, genetic variants in COX-2 and prostate cancer. J Nutrigenet Nutrigenomics 2: 149-158, 2009.

26. Kang KS, Wang P, Yamabe N, Fukui M, Jay T and Zhu BT: Docosahexaenoic acid induces apoptosis in MCF-7 cells in vitro and in vivo via reactive oxygen species formation and caspase 8 activation. PLoS One 5: e10296, 2010.

27. Bougnoux P, Hajjaji N, Ferrasson MN, Giraudeau B, Couet C and Le Floch O: Improving outcome of chemotherapy of metastatic breast cancer by docosahexaenoic acid: A phase II trial. Br J Cancer 101: 1978-1985, 2009.

28. Dimri M, Bommi PV, Sahasrabuddhe AA, Khandekar JD and Dimri GP: Dietary omega-3 polyunsaturated fatty acids suppress expression of EZH2 in breast cancer cells. Carcinogenesis 31: 489-495, 2010

29. Rose DP and Connolly JM: Regulation of tumor angiogenesis by dietary fatty acids and eicosanoids. Nutr Cancer 37: 119-127, 2000.

30. Altenburg JD and Siddiqui RA: Omega-3 polyunsaturated fatty acids down-modulate CXCR 4 expression and function in MDA-MB-231 breast cancer cells. Mol Cancer Res 7: 1013-1020, 2009. 